International Journal of Bifurcation and Chaos, Vol. 19, No. 8 (2009) 2621-2636

(c) World Scientific Publishing Company

\title{
DISSIPATIVE SOLITONS: PRESENT UNDERSTANDING, APPLICATIONS AND NEW DEVELOPMENTS
}

\author{
NAIL AKHMEDIEV and ADRIAN ANKIEWICZ \\ Optical Sciences Group, \\ Research School of Physical Sciences and Engineering, \\ The Australian National University, Canberra ACT 0200, Australia \\ JOSÉ-MARÍA SOTO-CRESPO \\ Instituto de Óptica, C.S.I.C., \\ Serrano 121, 28006 Madrid, Spain \\ PHILIPPE GRELU \\ Institut Carnot de Bourgogne, UMR 5209 CNRS, \\ Faculté des Sciences Mirande, \\ Avenue Savary, BP 47870, 21078 Dijon Cedex, France
}

Received April 9, 2008; Revised October 2, 2008

\begin{abstract}
Dissipative solitons form a new paradigm for the investigation of phenomena involving stable structures in nonlinear systems far from equilibrium. Basic principles can be applied to a wide range of phenomena in science. Recent results involving solitons and soliton complexes of the complex cubic-quintic Ginzburg-Landau equation are presented.
\end{abstract}

Keywords: Dissipative solitons; systems far from equilibrium; nonlinear dynamics.

\section{Introduction}

The concept of the dissipative soliton has received wide recognition in optics (physics in general), biology and medicine [Akhmediev \& Ankiewicz, 2005, 2008]. This concept allows many diverse phenomena in nature to be understood and studied in a simple and systematic way. It represents a unifying notion in science, since it stresses common features in a variety of complicated behaviors of self-localized formations. The most prominent feature of dissipative solitons is that they exist only when there is a continuous energy supply from an external source. This energy has to be dissipated in the medium where solitons are found. Thus, their formation requires a balance between the energy supplied and lost. This balance has to be exact in order to prevent overheating or cooling down of the system and the subsequent disappearance of the soliton. The exact balance is provided by the highly involved nonlinear dynamics in the system, which implies that dissipative solitons have to be self-organized. Their shape, amplitude, width and all other parameters are unique for given external conditions. Dissipative solitons can be natural, i.e. they can exist in nature as a result of a relaxation to a localized formation under the influence of solar heating and dissipation, or they can be artificial objects created by scientists. In the latter case, they can be used in modern technology.

A common misconception concerning dissipative systems is that they can only have losses and thus only decaying solutions. The name "Dissipative system" was coined by [Nikolis \& Prigogine, 1977] for systems considered in nonequilibrium 
thermodynamics. These systems are not isolated, but are kept in contact with an external source that provides energy for the smaller subsystem. Thus dissipation is essential for the transfer of pumped energy to a "cooler". Hence the notion "dissipative system" is more complicated. It assumes that there is also an energy supply part, rather than just losses. Localized structures in these systems, i.e. "dissipative solitons" have their own right to be an established scientific keyword.

Examples of dissipative solitons include [Akhmediev \& Ankiewicz, 2005, 2008] ultrashort pulses from passively mode-locked lasers, nerve pulses, localized formations in reactiondiffusion systems, vegetation clustering in arid lands, Bose-Einstein condensates in cold atoms, wave phenomena in neuron networks, spiral waves in weakly-excitable media and traveling waves in cortical networks. This list is far from being complete. The variety of systems that admit dissipative solitons is enormous. So is the variety of mathematical equations they satisfy. Nevertheless, they all have a common feature: they only "live" in the presence of a continuous energy supply from an external source. Thus, they can be studied using certain common rules and principles.

Passively mode-locked lasers generate dissipative solitons [Renninger et al., 2008]. This occurs naturally when the cavity is designed to have better finesse at higher intensities of the optical field than at lower ones. There is a variety of lasers, including solid-state, dye, semiconductor and fiber examples [Haus, 2000; Salhi et al., 2008], with a large number of designs of passive mode-locking mechanisms. The pulses that are generated in this way are perfect examples of self-organized structures. The temporal shape and spectrum of the pulses depend on several cavity parameters and can be varied over a wide range. Pulses can take sech-profiles, Gaussian profiles, almost rectangular "flat-top" profiles, etc. Dissipative solitons can be generated for both anomalous and normal average cavity dispersions [Chong et al., 2008]. It turns out that normal cavity dispersion may lead to extremely high energy output pulses [Fernandez et al., 2004; Wise et al., 2008]. This important application of dissipative solitons is related to "dissipative soliton resonances", which will be described later in this paper.

One of the most popular approaches to study dissipative solitons is based on the complex cubicquintic Ginzburg-Landau equation (CGLE). The CGLE is a generic equation that describes systems near subcritical bifurcations [van Saarlos \& Hohenberg, 1990; Deissler \& Brand, 1994]. This equation applies to a large variety of phenomena in science. In fact, the scope of this knowledge has been called "the world of the complex Ginzburg-Landau equation" [Aranson \& Kramer, 2002]. Even the small part of this realm involving only localized solutions is a world by itself. The Ginzburg-Landau equation, taking terms into account up to cubic, presents a vast diversity of such solutions, but the realm of solutions becomes infinitely richer when we take into account quintic terms in the equation. Complications arise from both physical and mathematical points of view. Despite these profound intricacies, we can save this sphere of knowledge from being esoteric by applying basic principles of dissipative solitons.

The model based on the CGLE includes cubic and quintic nonlinearities of both dispersive and dissipative types. The normalized master equation in the (1+1)-dimensional case reads [Akhmediev \& Ankiewicz, 1997; Aranson \& Kramer, 2002]:

$$
\begin{aligned}
i \psi_{z} & +\frac{D}{2} \psi_{t t}+|\psi|^{2} \psi+\nu|\psi|^{4} \psi \\
& =i \delta \psi+i \epsilon|\psi|^{2} \psi+i \beta \psi_{t t}+i \mu|\psi|^{4} \psi .
\end{aligned}
$$

The optical field envelope, $\psi$ in Eq. (1), is a complex function of two real variables, i.e. $\psi=\psi(t, z)$, where $t$ is the retarded time in the frame moving with the pulse, and $z$ is the propagation distance. The lefthand side of Eq. (1) contains the conservative terms with the coefficients $D$ and $\nu$. Here, $D$ is the dispersion coefficient, which is positive (negative) in the anomalous (normal) propagation regime, while $\nu$ represents a higher-order Kerr nonlinearity. The right-hand side of Eq. (1) includes all dissipative terms. The coefficients $\delta, \epsilon, \beta$ and $\mu$ control the averaged linear loss in the cavity (when negative), nonlinear gain (if positive), spectral filtering or gain dispersion (if positive), and saturation of the nonlinear gain (if negative), respectively. This master equation can be applied to the modeling of passively mode-locked lasers in the short pulse regime of operation. In principle, a continuous model should be valid when changes in the pulse during a round trip are relatively small, but we have determined that the validity of the predicted dynamics can be qualitatively good even in cases of stronger internal pulse dynamics. The laser is assumed to be in a stationary regime, so that all transient effects which occur after starting the laser have vanished. Dissipative terms describe the averaged gain and loss processes taking 
place when the pulse moves in the cavity. Higherorder dissipative terms describe the cumulative nonlinear transmission characteristics of the cavity, including the passive mode-locking mechanism.

The cubic-quintic complex CGLE allows the prediction of highly complicated pulse dynamics, such as "exploding solitons" [Cundiff et al., 2002]. Finding qualitatively new solutions of the CGLE significantly broadens the "world of the Ginzburg-Landau equation" [Aranson \& Kramer, 2002] and, indeed, these solutions can be useful in all areas of science where this universal equation can be applied. For instance, preliminary modeling is essential to provide rough estimates for the range of parameters that would lead to the generation of pulses with the highest possible energy from a single laser oscillator.

The cubic-quintic CGLE admits a wide variety of soliton solutions [Akhmediev et al., 2001; Soto-Crespo et al., 1997; Soto-Crespo et al., 2001; Descalzi \& Brand, 2005; Descalzi et al., 2005]. The presence of six parameters in the equation makes it difficult to find all of these solutions at once. Our primary task is to find a region of parameters of physical significance where stationary stable solitons do exist [Descalzi, 2003]. Once one stable localized solution is found for a given set of equation parameters, it can serve as the initial condition for finding solutions at other nearby values of the parameters. By moving slowly in the parameter space, we are able to determine regions of soliton existence in a relatively easy way. Our present technique exclusively produces robust stable solitons. In general, we fix four of the parameters, for instance $\mu, \nu, \delta, \beta$, and change $D$ and $\epsilon$ when looking for stable localized solutions. Indeed, dispersion and gain can be quite easily controlled in any passively mode-locked laser, whether it be a solid-state or a fiber device.

An important characteristic of the output pulse is its total energy $Q$, given by the integral of $|\psi|^{2}$ over $t: Q(z)=\int_{-\infty}^{\infty}|\psi(t, z)|^{2} d t$. For a dissipative system, the energy is not conserved, but it evolves in accordance with a balance equation [Akhmediev \& Ankiewicz, 1997]. If the solution stays localized, the energy can evolve but it remains finite. Furthermore, when a stationary solution is reached, the energy, $Q$, converges to a constant value.

\section{Dissipative Solitons and Antisolitons}

When we change the parameters of a system, we usually expect that the properties of a soliton will change continuously, i.e. that two stationary solitons belonging to two different points in the parameter space will be gradually transformed into each other. However, it happens that this is not always the case. There are two distinctively different areas where dissipative solitons exist. There is no way of continuously transforming a soliton from one region to the other.

Figure 1 shows some regions in the $(\mu, \epsilon)$ plane where stationary localized solutions can be found. Indeed, there are two separate regions in the parameter space where distinct types of dissipative solitons exist. The red regions correspond to stable stationary solitons, while the gray region corresponds to exploding solitons [Soto-Crespo et al., 2000; Akhmediev et al., 2001; Akhmediev \& SotoCrespo, 2003]. The latter region indicates nothing other than the zone where the upper branch solutions become unstable. The soliton properties of the upper branch are in marked contrast to the properties of solitons in the lower branch, as explained below. Figure 2 represents the results of numerical simulations in the plane $(\nu, \epsilon)$. Again, red regions are for stable solitons while the gray region is for exploding solitons. We note that the parameter $\beta$ is not the same in the two plots. This is essential for the two regions to be revealed at the same plot. It would be difficult to find the set of parameters

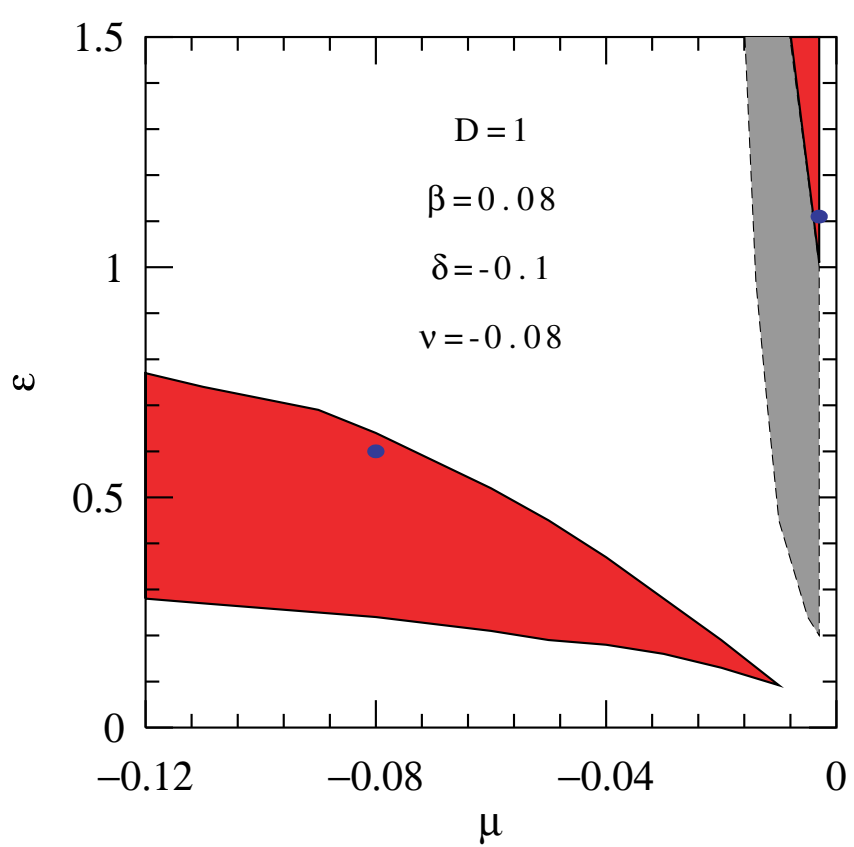

Fig. 1. Regions of existence of the two types of solitons (red) in the $(\mu, \epsilon)$ plane. The two separate regions are quite distinct. Parameters are shown in the plot. The gray region corresponds to exploding solitons. 


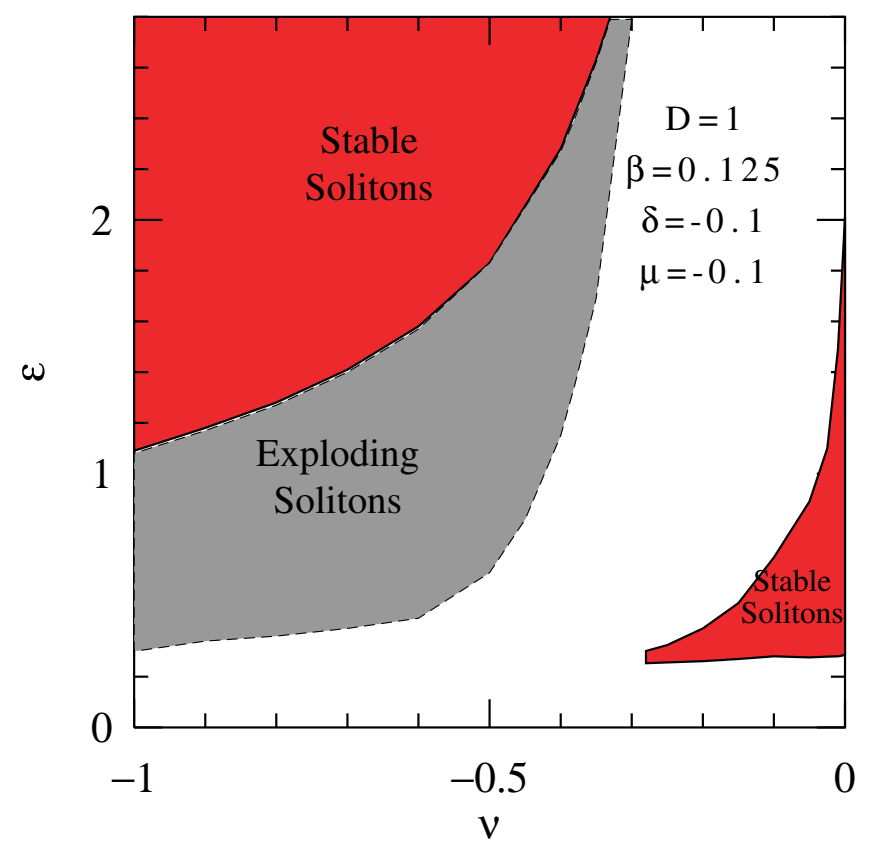

Fig. 2. Regions of existence of the two types of solitons (red) in the $(\nu, \epsilon)$ plane. Parameters are shown in the plot. The gray region corresponds to exploding solitons.

which would allow us to create a 3 -D plot that simultaneously shows the two regions.

Two illustrative examples for the field amplitude and phase profile of the solutions for each region of soliton existence are presented in Fig. 3. Solid lines represent the field amplitude of the solitons while the dashed lines are their phase profiles. The upper curves (a) in Fig. 3 correspond to the upper blue point in Fig. 1 while the lower curves (b) correspond to the lower blue point. There are some obvious differences in the energies, widths and amplitudes of the two solitons. However, the greatest qualitative difference is in the chirp. Clearly, the phase profiles show that the chirps are of opposite sign. Due to this difference, the energy flows from the inside to the outside of the pulse in the first case while it flows inwards in the second case. The phase profile is not exactly parabolic, of course, but it is clear from Fig. 3 that the effective parabolic chirp coefficients $\left(c_{0}\right)$ in the solution $\psi \sim \exp \left(+i c_{0} z^{2}\right)$ are opposite in sign, and that the field amplitude in (a) is much greater than that in (b).

In Fig. 2, we can say that we have $c_{0}>0$ in the low energy (right-hand-corner) region, while we find $c_{0}<0$ in the upper left corner (high energy). Approximate semi-analytic results obtained from a reduced model [Ankiewicz et al., 2007] predicted this behavior. The different signs appearing for each

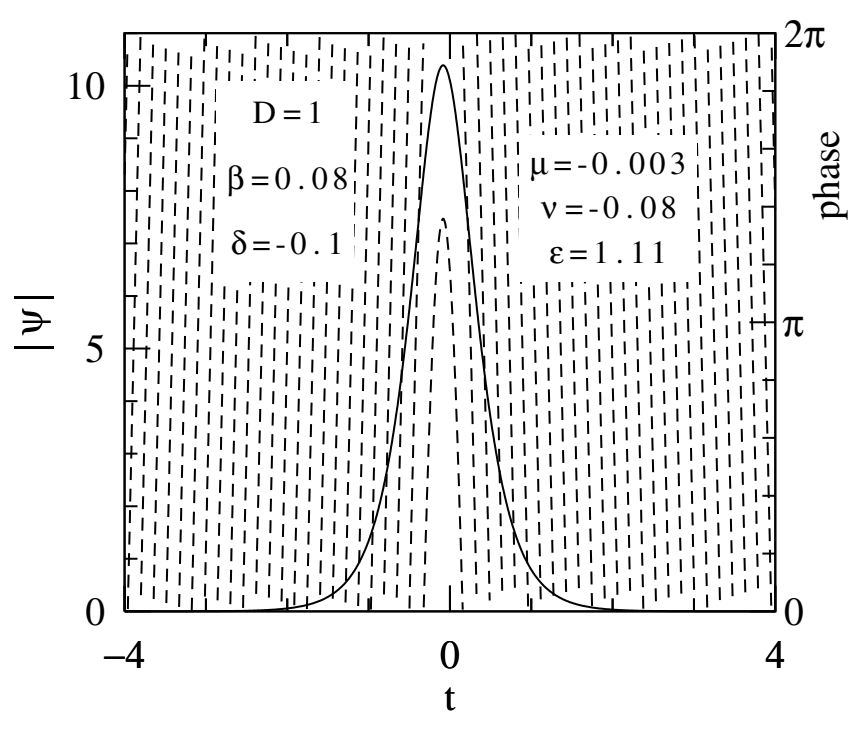

(a)

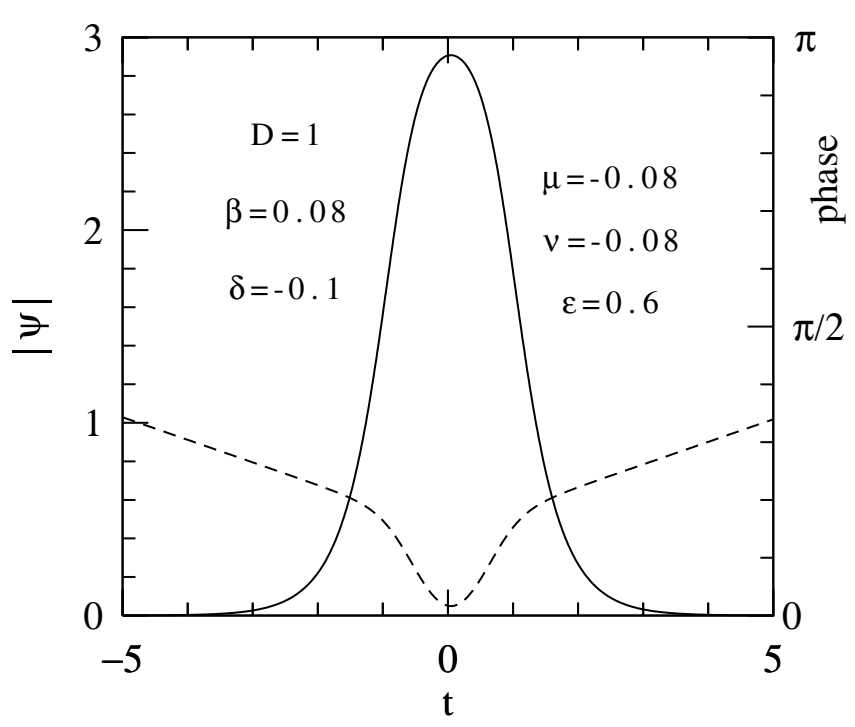

(b)

Fig. 3. Soliton amplitude (solid lines) and phase (dashed lines) profiles from two examples in (a) upper (antisoliton) and (b) lower (soliton) regions in Fig. 1. They are marked by blue thick dots in Fig. 1. The vertical phase scale is taken in the interval from 0 to $2 \pi$. Thus, the phase in (a) changes rapidly.

region mean that the phase profile across the soliton is concave up in one case, and concave down in the other. Hence they can be designated as "solitons" and "antisolitons" [Ankiewicz et al., 2007].

This qualitative difference can be made clearer if we plot the distribution of energy $P$ generated and dissipated inside of the soliton. These curves are shown in Fig. 4. We can see clearly that, in the first 


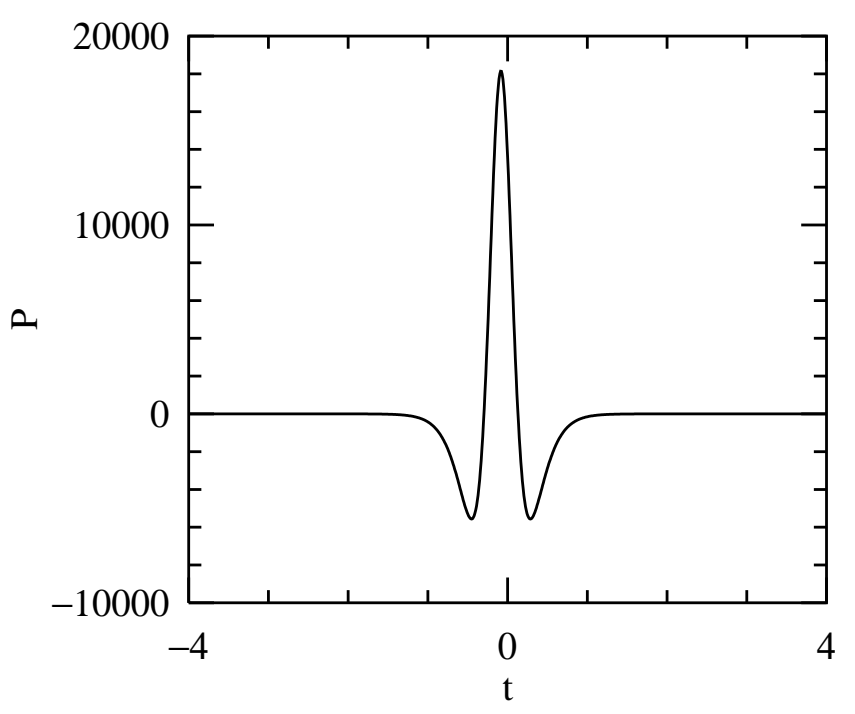

(a)

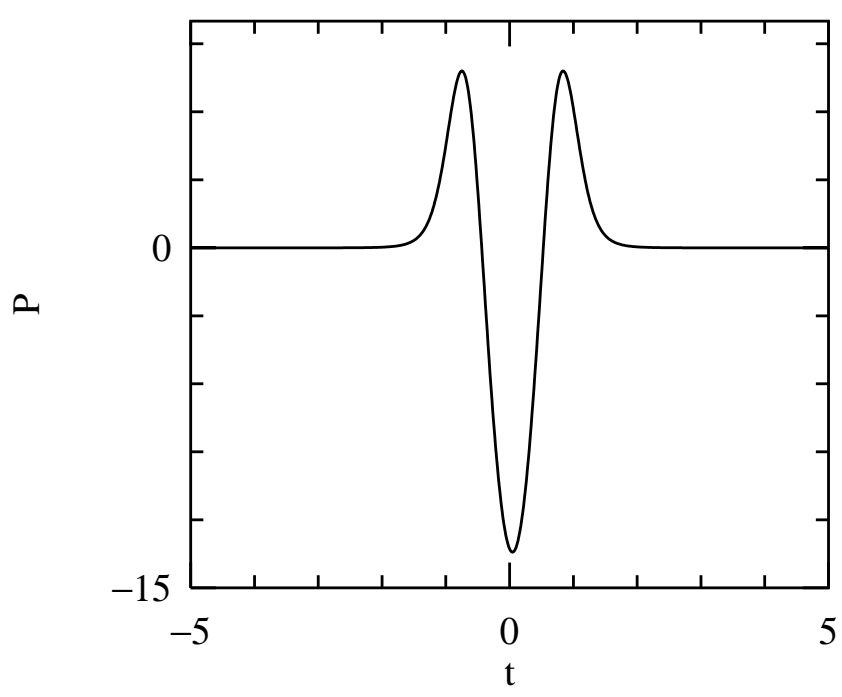

(b)

Fig. 4. Energy generation, $P$, inside the two types of solitons. In (a), the energy is generated in the middle of the soliton and is dissipated in the tails while in (b), it is generated in the tails and dissipated in the center.

case, the energy generation is positive in the middle of the soliton and negative in the wings, while in the second case, the energy is generated in the tails of the soliton and dissipated in the middle. Thus, there is a fundamental qualitative difference between the two types of solitons. One type cannot be transformed into the other with a continuous change of parameters. There is even a more significant difference between them: the upper branch admits the socalled "dissipative soliton resonance", as explained below.

\section{Dissipative Soliton Resonances}

There is a region in the parameter space where stable dissipative solitons have energies much higher than those observed before. In fact, the energy can reach extremely high values [Akhmediev et al., 2008]. This phenomenon can be called "dissipative soliton resonance". When the quintic nonlinearity is positive $(\nu>0)$, solitons can be observed for both anomalous $(D>0)$ and normal $(D<0)$ dispersion regimes. Figure 5 shows a region of existence of dissipative solitons for this case. When $\nu$ is negative, solitons may exist but in a significantly narrower region of values of the parameter space.

Examples of dissipative soliton spectra and pulse shapes are presented in Figs. 6 and 7. They correspond to the thick dots in Fig. 5, using the same color code. Soliton shapes and spectra change with a change in the dispersion, $D$, and the cubic gain parameter, $\epsilon$. In particular, the soliton spectrum changes dramatically with a change in $D$. When increasing the absolute value of $D$ in the region of existence with negative $D$, the spectrum becomes much wider and acquires an approximately rectangular shape, with two or three local maxima. The qualitative shape of the spectrum is defined mainly by $D$ but its spectral width depends predominantly on the cubic gain parameter $\epsilon$. Figure 6 shows that for fixed $\epsilon$, the width of the soliton spectrum is almost constant. However, the spectral

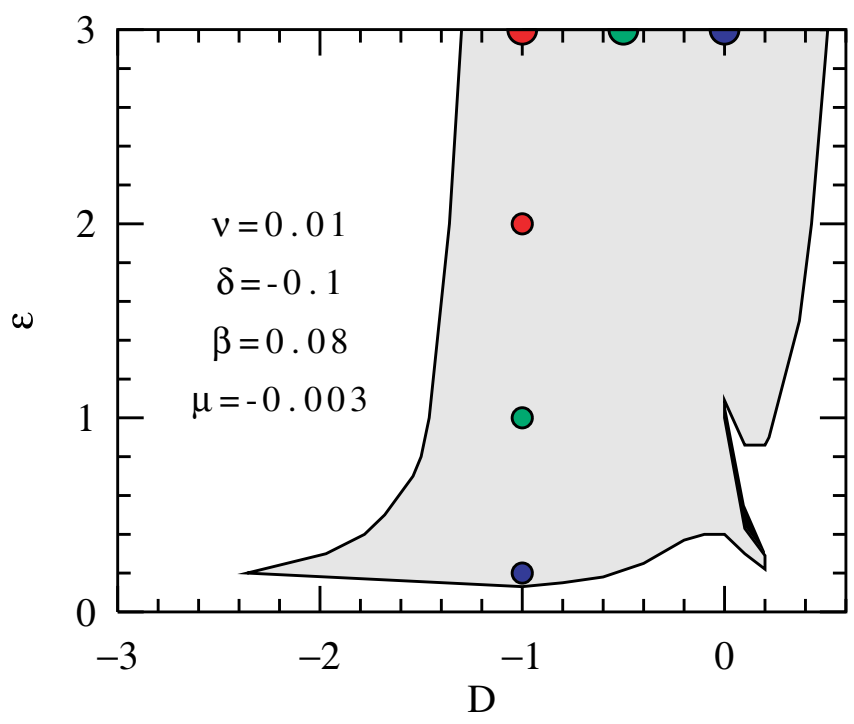

Fig. 5. Region of existence of dissipative solitons (gray) that extends from positive to negative values of dispersion, $D$. Solid dots correspond to the examples of soliton profiles and spectra given below. 


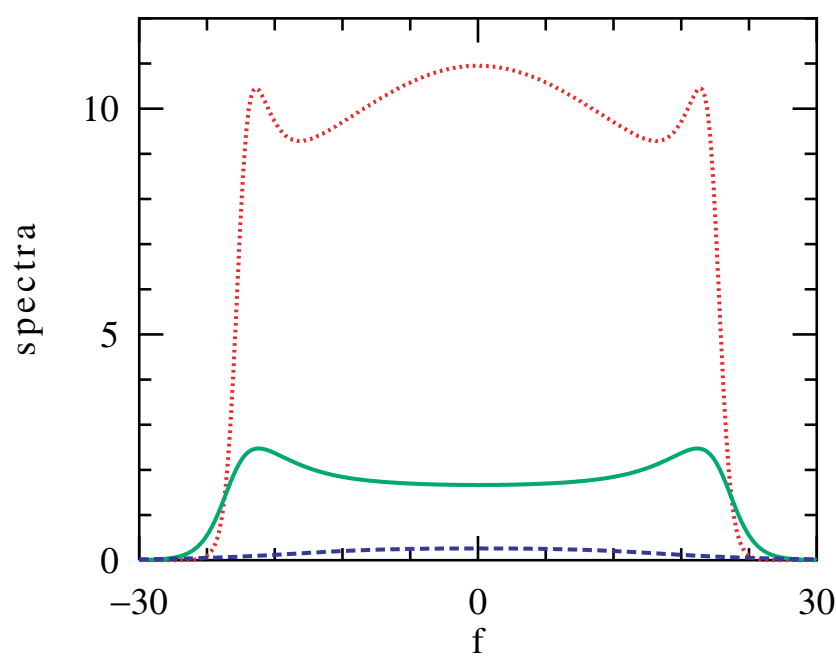

(a)

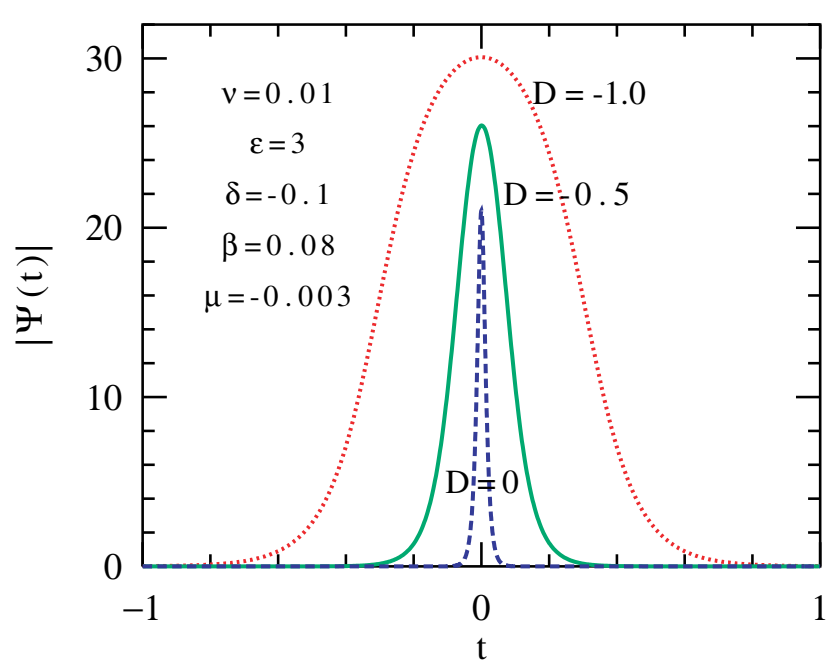

(b)

Fig. 6. (a) Soliton spectra and (b) pulse profiles for the set of parameters shown in (b). The curves correspond to the thick dots along the upper horizontal line of the plot in Fig. 5.

intensity depends strongly on the dispersion parameter, so that the total energy of the pulse increases when the $D$-value shifts towards the left-hand side of the region of existence.

Figure 7 shows the dependence of the spectra and pulse profiles on the cubic gain parameter, $\epsilon$, when $D=-1$. With an increase in $\epsilon$, the pulse becomes narrower and higher while the spectrum becomes wider, keeping its roughly rectangular profile. An interesting feature of the spectra at this value of $D$ is that they all have three maxima rather than two. Moving to even more negative values of $D$, the central maximum in the spectra increases considerably. The pulses shown in Figs. 6 and 7

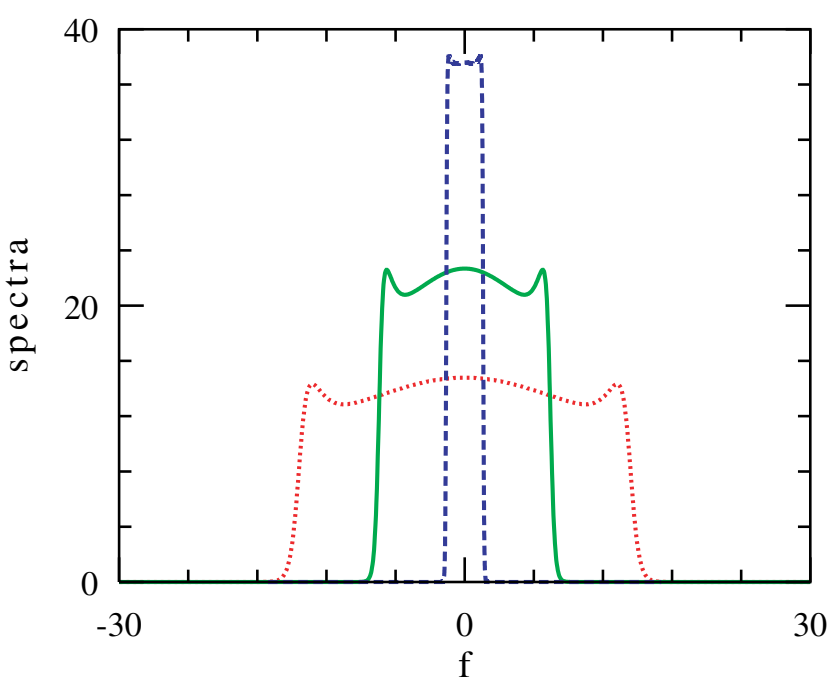

(a)

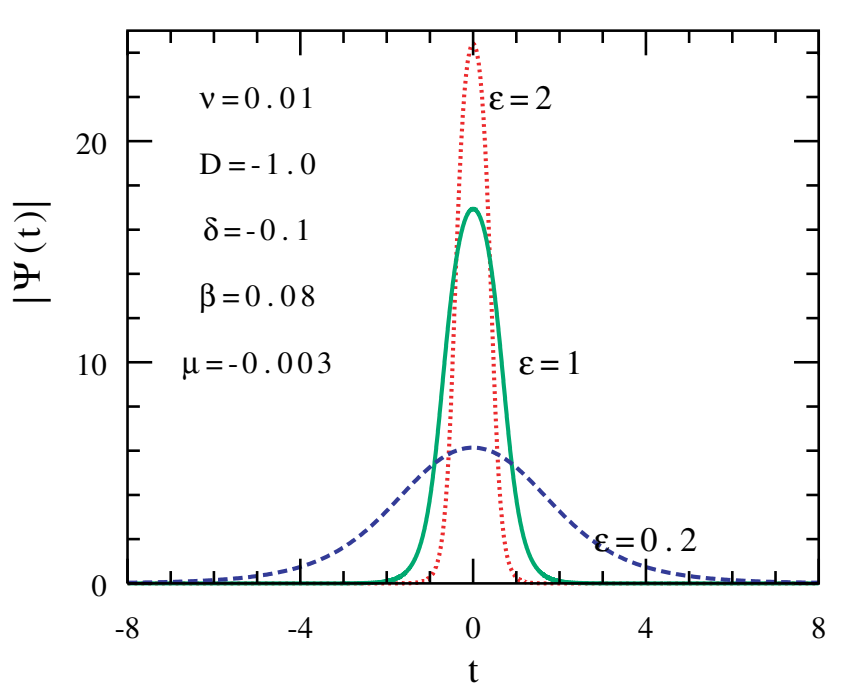

(b)

Fig. 7. (a) Soliton spectra and (b) pulse profiles for the set of parameters shown inside the plots. The curves correspond to the solid heavy dots along the vertical line $D=-1$ in Fig. 5 . Increasing the cubic gain results in shorter pulses with wider spectrum and slightly higher energy.

are clearly far from being Fourier-transform limited. However, the pulse duration can be significantly shortened by de-chirping it in a dispersion compensation line. We verified numerically that close-totransform-limited, ultra-short pulses, can thus be obtained.

The most remarkable feature of dissipative solitons in the normal dispersion regime is that their energy $Q$ can grow indefinitely when the absolute value of $D$ increases. Figure 8 shows the energy, $Q$, versus the dispersion $D$ for three values of $\epsilon$. 


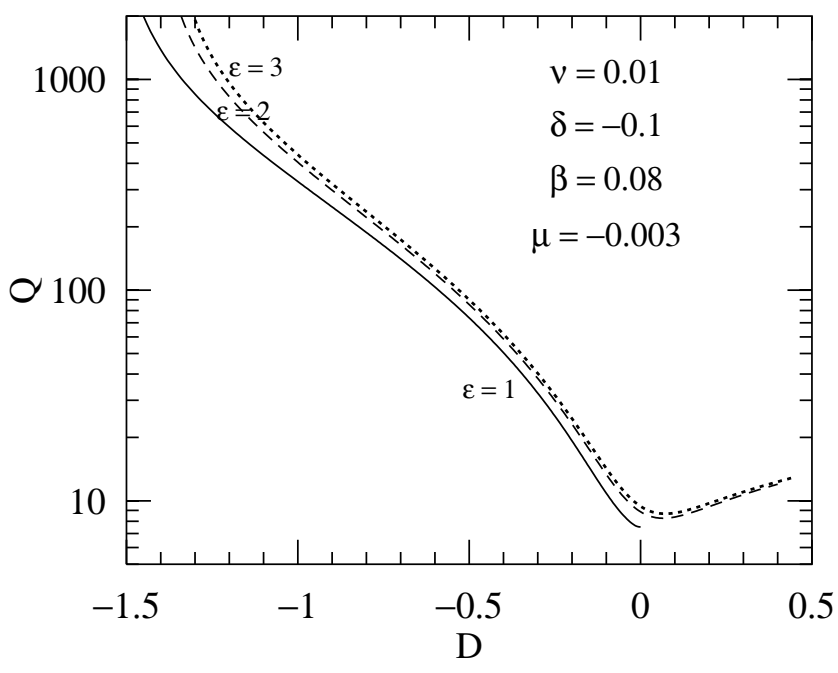

Fig. 8. Soliton energy $Q$ versus dispersion parameter $D$, for three $\epsilon$ values.

This figure shows clearly that the energy $Q$ depends strongly on the dispersion $D$. In fact, the energy increases to infinitely large values when $D$ reaches the edge of stability on the left-hand side of the region of existence in Fig. 5. This result shows that the only practical limit to increasing the values of the pulse energy in the normal dispersion regime is the pump power and the stability of the pulses with respect to higher-order terms which are not included in our model.

The region of existence of new solitons can be presented most efficiently in a two-dimensional plot such as that in Fig. 5. The four other parameters can be found as a result of a thorough search in the parameter space by selecting the values that provide the highest energy. Without prior knowledge of at least some of the regions of soliton existence [Soto-Crespo et al., 1997], the search could have required lengthy numerical simulations. In particular, we found that the higher-order nonlinearity $\nu$ should be positive. When $\nu$ is negative, the region of existence of normal dispersion solitons shrinks significantly (see Fig. 8 of [Soto-Crespo et al., 1997]). In particular, for the chosen values of $\beta$ and $\mu$, highenergy solitons cease to exist at negative $\nu$.

Figure 9 shows a region of existence of dissipative solitons in the case of negative $\nu$. As we can see, the main difference between this region and the one shown in Fig. 5 is the absence of solitons in the negative dispersion area. The energies of solitons in this positive $D$ area are reduced and they do not show any relevant increase at the edges of the region. Instead, on the left-hand side edge of the

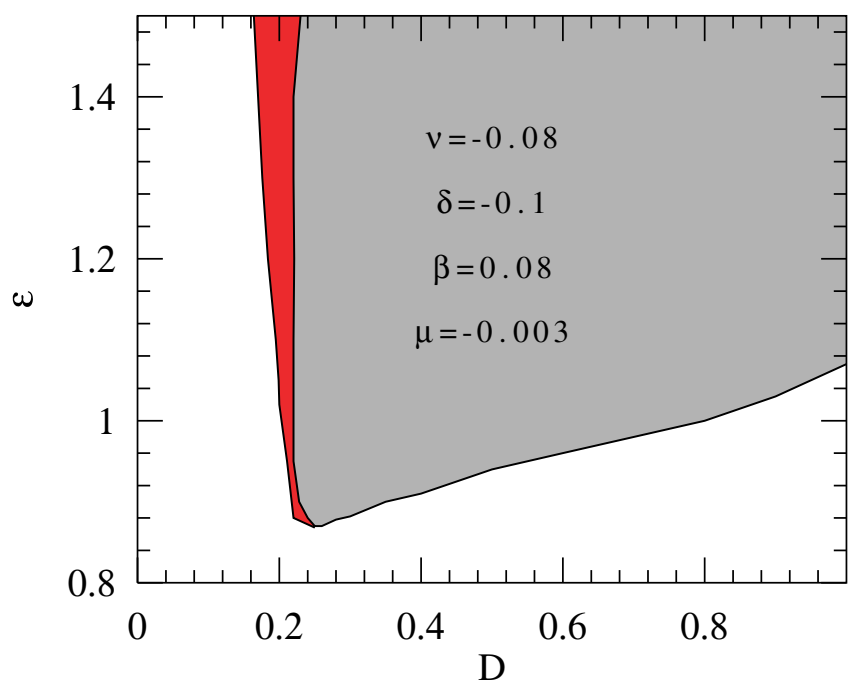

Fig. 9. Region of existence of dissipative solitons in the case of negative $\nu$. The red strip on the left-hand side border shows a region of existence of pulsating solitons.

region, we observed a strip of pulsating solitons. On decreasing the value of $D$ further, no stable localized solution was found. We should emphasize that, for different sets of equation parameters, dissipative solitons were found in the normal (negative) dispersion regime for negative $\nu$ [Soto-Crespo et al., 1997], but, in that case, the properties of pulses, such as phase profiles and energies, differ greatly from the those of the "antisolitons" presented above.

This example shows once again that the choice of parameters of the master equation is the key for the observation of high-energy solitons. As we have six parameters in Eq. (1), the search for other regions of the parameter space where high-energy solitons can be found is a tedious task. However, these studies can provide us with an efficient guideline for experimental research, so that these efforts are worthwhile.

These observations are potentially useful for many applications where the CGLE is used [Aranson \& Kramer, 2002]. In particular, the results can be highly relevant for further development of highpower passively mode-locked lasers. Recently, Fernandez et al. may have observed similar high-energy dissipative solitons in their experiment with a Kerrlens mode-locked Ti:sapphire laser. The direct evidence for this statement is the optical spectrum of Fig. 3(a) in [Fernandez et al., 2004], which is very similar to the spectra presented in Figs. 6 and 7, apart from the asymmetry which originates from higher-order dispersion. The pulse in the experiment was strongly chirped and of high power, as 
follows from our numerical study. Clearly, further common theoretical and experimental work in this direction will allow us to optimize the parameters and generate pulses with exceptionally high energy without additional external amplification.

\section{Dissipative Soliton Interactions}

Dissipative systems admit robust soliton pairs. Since their stable existence was first predicted [Akhmediev et al., 1997] in systems governed by the complex Ginzburg-Landau equation, they have been experimentally observed on various occasions in fiber lasers [Grelu et al., 2002]. When they exist, dissipative solitons usually have a fixed profile. This fact allows us to describe the interaction between two of them using just two variables, namely the separation, $\rho$, and the phase difference, $\theta$, between the two pulses. Therefore the dynamics of a pair of solitons can be usually described without ambiguity in a two-dimensional phase space that is usually called the "interaction plane" [Akhmediev et al., 1997]. However, this is not always the case [SotoCrespo et al., 2007].

Even single solitons in dissipative systems can have complicated behaviors. They can be pulsating, creeping or exploding [Soto-Crespo et al., 2000] and exhibit many other types of dynamics [Akhmediev et al., 2001]. These are all determined by the parameters of the system. Being equipped with this knowledge, one would expect that soliton pairs could also show complicated behavior. In particular, it has been found that a soliton pair can pulsate or evolve chaotically [Soto-Crespo et al., 2007]. Systems with an infinite number of degrees of freedom are likely to exhibit a wide range of complicated dynamics, and the reduction to simpler systems with a twodimensional phase space cannot be applied as a general rule. At least for some regions of the parameter space, more degrees of freedom need to be considered to understand the dynamics. In these cases, the interaction plane is clearly not adequate to describe the dynamics of pairs in its full complexity.

There are complicated cases where soliton pairs are oscillating in time, either periodically or chaotically [Soto-Crespo et al., 2007]. In each case, the soliton pair exists indefinitely in time as a bounded, localized two-soliton solution, thus manifesting stability. Three new types of bound states can be distinguished: the "vibrating soliton pair" (VSP), "shaking soliton pair" (SSP) and "mixed soliton pair" (MSP). The VSP shows simple oscillations of the soliton pair variables. These oscillations can be considered as limit cycles of our dynamical system with an infinite number of degrees of freedom (see discussions on this subject in Chapter 1 in [Akhmediev \& Ankiewicz, 2005]). The SSP is essentially a strange attractor. Its behavior is somewhat similar to that of a single exploding soliton [Soto-Crespo et al., 2000]. There are also transitions between these various propagation regimes which occur when the parameters of the system are changed, and these manifest themselves as bifurcations in the soliton pair dynamics. An interesting feature of this complex dynamics is that it is specific for the soliton pair - each soliton forming the pair has perfectly stable stationary behavior, when isolated, for the same set of the equation parameters. The third new type of soliton pair comprises MSPs, which consist of two different objects, viz. a composite pulse and a plain pulse. Although their nonlinear propagation constants are different, they can still form stable pairs having a periodic evolution of their phase difference and a fixed separation [Soto-Crespo et al., 2007].

When the number of interacting solitons exceeds two, the variety of possible dynamics becomes enormous, and it becomes difficult to construct interaction planes due to the increased number of the effective variables which are the relative phases and temporal separations between consecutive solitons. However, complexes of more than two solitons represent an important area for future research, since they have been found experimentally in fiber lasers [Grelu \& Soto-Crespo, 2004] and could find application in the design of specific ultrafast pulse-packet sources, as well in the control of optical data packets in telecommunication. The case of three interacting solitons has been addressed theoretically [Akhmediev et al., 2005], to explain the situations that have been unveiled experimentally, such as collisions of elastic-type between a soliton pair and a third moving soliton [Grelu \& Akhmediev, 2004; Oliver et al., 2004] and soliton triplet formation and dissociation. Stable soliton triplets can take numerous forms when nonidentical temporal spacing between successive solitons is allowed, as was deduced from experiments [Grelu et al., 2003]. Theoretical studies become more tractable in the case of identical spacing, and some classification becomes possible, such as the "cis" and "trans" isomers of a soliton triplet [Leblond et al., 2006].

However, the building block for understanding the formation of soliton complexes resides in the 
study of two interacting solitons. Hence, the recent discoveries of soliton pair dynamics are explained below.

\subsection{Vibrating soliton pairs}

Periodic orbits are stable robust formations which are limit cycles of the CGLE. As such, they are attractors of the nonlinear dynamical system. An example is shown in Fig. 10(a). Suppose that we use an initial condition in the form of two solitons separated by a finite distance. When starting from an arbitrary point in the interaction plane, located inside or outside of the limit cycle in Fig. 10(a), the trajectory converges to this limit cycle, rather than to a fixed point. Moreover, when the initial conditions are not exactly two solitons at a fixed

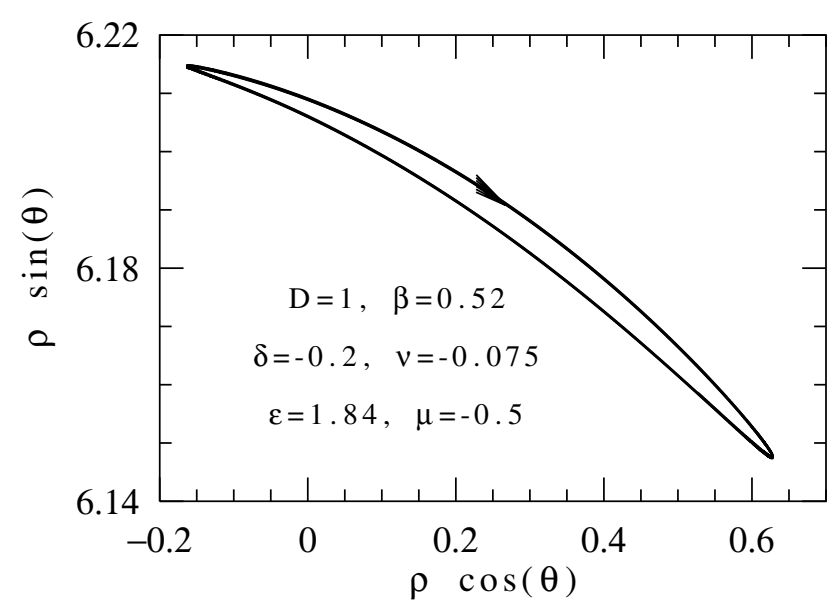

(a)

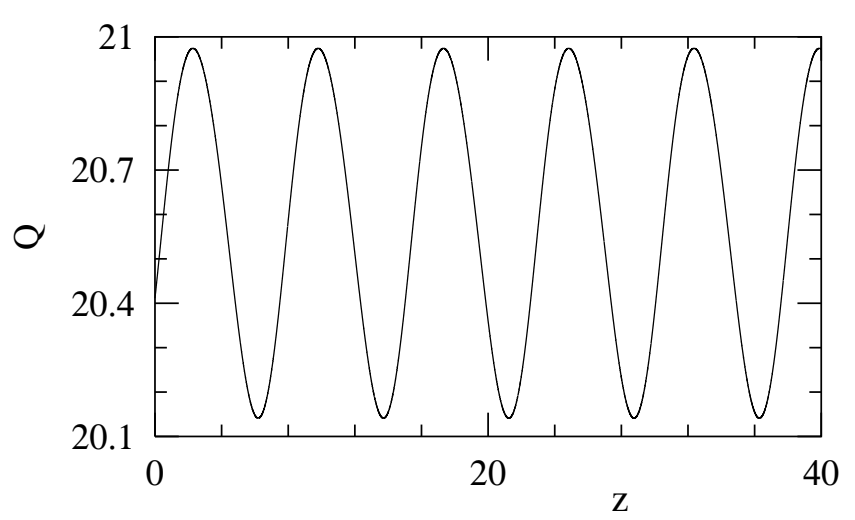

(b)

Fig. 10. (a) Limit cycle on the interaction plane that corresponds to a VSP. The arrow indicates the clockwise rotation of the trajectory. (b) Periodic evolution of the energy, $Q$, for the same case. The equation parameters used in the simulation are written in (a). distance, but two pulses with a shape that only roughly resembles solitons, the trajectory also converges to the same limit cycle. The early part of the trajectory in the phase space depends on the initial condition and can be rather complicated. Thus, all such parts of the trajectories have been removed from Fig. 10(a) for the sake of clarity. Only the limit cycle, which is the final part of stable evolution, is shown. It is represented by the solid curve in the figure. The arrow shows the direction of motion in the interaction plane.

This type of solution is called a "vibrating soliton pair" or VSP. The existence of vibrating soliton pairs was found experimentally in a mode-locked fiber laser and confirmed numerically from a propagation model including discrete elements [Grapinet \& Grelu, 2006], and was finally unveiled from the dynamics of a single CGLE, as presented above [Soto-Crespo et al., 2007].

For the given sets of the system parameters, only two types of solutions exist - single stationary solitons and vibrating pairs. Soliton pairs with fixed distance and phase difference do not exist for this set of parameters. Thus, any initial condition which is a bound pair of two pulses located in the basin of attraction will give birth to a VSP, rather than to a stationary soliton pair.

The trajectory shown in Fig. 10(a) is noticeably asymmetric relative to the vertical line which corresponds to a phase difference of $\pi / 2$. The distance, $\rho$, between the two maxima of the pulses oscillates, as does the phase difference. This set of two pulses has a finite velocity, moving towards the right (positive $z$ direction). The peak amplitude of the pulse on the right-hand side is slightly larger than that of the pulse on the left. This asymmetry comes from the nonsymmetric phase relationship between the two pulses. However, due to the $t \leftrightarrow-t$ symmetry of the CGLE, there is always the mirror-image VSP solution moving towards the left and having a phase difference close to $-\pi / 2$. The periodic evolution of the energy, $Q$, versus $z$ is shown in Fig. 10(b). This single periodic curve is very close to being harmonic.

The pulse profile evolution is shown in Fig. 11 for the same propagation distance as in Fig. 10(b). The periodic evolution is better observed from the slopes of the two solitons than at the maxima. The reason why the vibration appears with a small amplitude in this diagram is mainly due to the fact that, in the example chosen, it is mostly the relative phase which oscillates, while the relative separation 


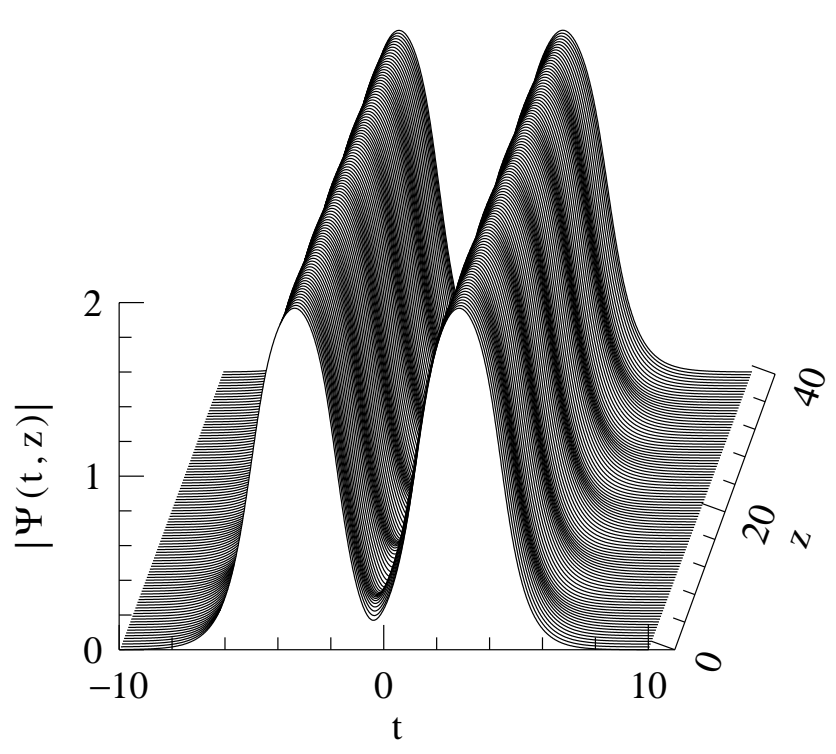

Fig. 11. Evolution of the pulse profile for the vibrating soliton pair (VSP). Parameters of the simulation are the same as in Fig. 10.

oscillates only by $0.5 \%$, as can be seen in Fig. 10(a). This solution can be viewed as the pulsating twosoliton generalization of a single pulsating soliton [Soto-Crespo et al., 2000; Akhmediev et al., 2001]. However, pulsations here are solely due to the interaction between the two solitons. Single pulsating solitons do not exist for the set of parameters chosen for these simulations. At the same time, two single pulsating solitons do not create a VSP. The pulsations of both solitons cause them to merge into one. Thus, we can consider a VSP to be a new object in the family of localized solutions of the CGLE.

\subsection{Shaking soliton pairs}

A second object that was recently found numerically [Soto-Crespo et al., 2007] is a "shaking soliton pair" (SSP). Its dynamics demonstrates the presence of chaotic effects in the evolution of soliton pairs. These are stationary pairs that have an intrinsic instability of an oscillatory type. The pair can be represented on an interaction plane as a fixed point which is an unstable-stable focus. An example of such a point is shown on the interaction plane in Fig. 12. The trajectory that describes the evolution of this pair is a spiral that winds out off the focus, makes a loop and winds in back to the initial point, thus repeating the cycle again and again. The cycles are similar to each other but are not exactly the same. For clarity, only one of the cycles is shown in Fig. 12. In a global evolution, each cycle is a

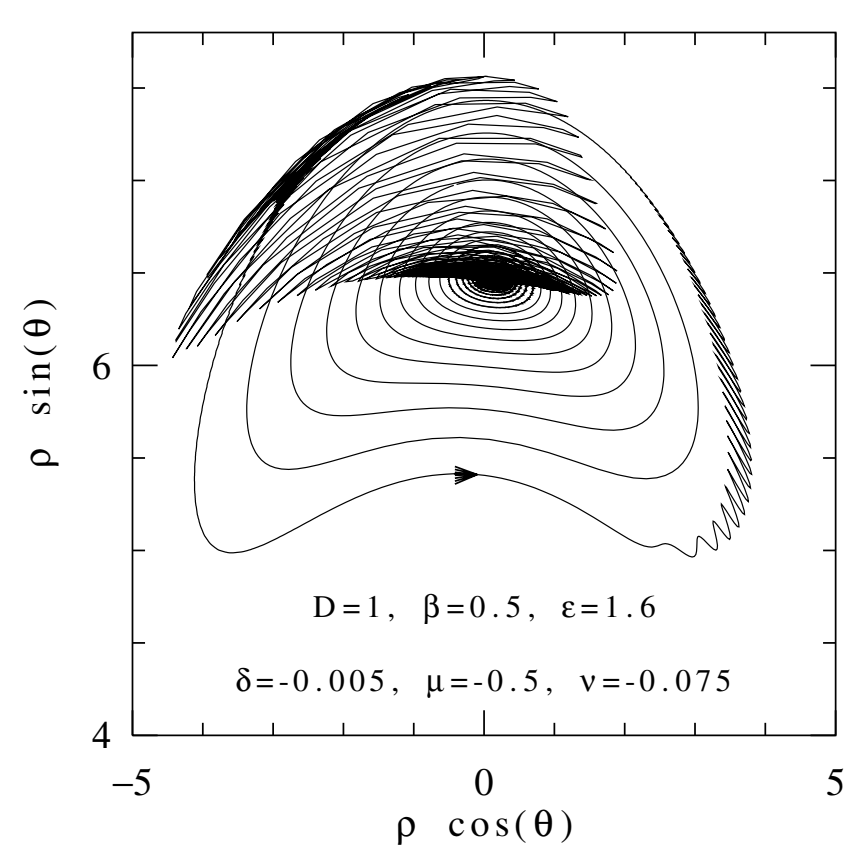

Fig. 12. Trajectory of the motion on the interaction plane for a soliton pair that is spontaneously shaken. The approach to the center and departure from it follow very different paths on the plane. Parameters of the simulation are written inside the figure.

homoclinic orbit returning back to the same point. The process of return is clearly seen in Fig. 12.

The center manifold of this dynamics is at least four-dimensional. The inward (outward) spiraling trajectory can be related to a fixed point with its corresponding linearized stability analysis providing two complex conjugate eigenvalues with negative (positive) real part. Thus, a complete description needs at least two pairs of complex eigenvalues. The trajectory in this reduced phase space escapes the fixed point in one two-dimensional subspace and returns to this point in another two-dimensional subspace.

The evolution of the total energy, $Q$, of the shaking pair is shown in Fig. 13. When the pair is in the nearly stationary part of the evolution, the energy appears to be constant. This corresponds to the fixed point in Fig. 12. In this example, the soliton pair spends most of its propagation time very close to this fixed point. On the other hand, when the pair is disturbed by the instability, the energy changes and evolves with the oscillations. Two shaking parts of the evolution are clearly seen in Fig. 13. When the instability is over, the energy returns to the same constant value as before. The cycles repeat indefinitely in ways that are similar, but not exactly 


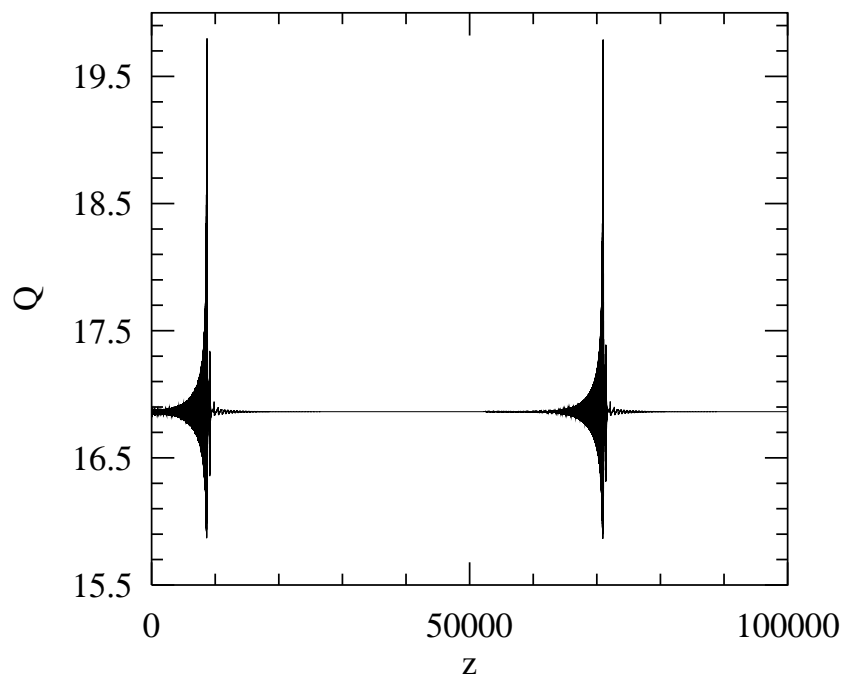

Fig. 13. Energy $Q$ versus $z$ for a soliton pair that is spontaneously shaken as an instability takes place. It corresponds to the same case as the one shown in Fig. 12.

the same. The evolution of the pulse profile during one cycle is illustrated in Fig. 14.

When changing the parameters of the system, the stationary part of the evolution may become shorter in comparison with the shaking part. Another plot for the energy $Q$ versus $z$ is shown in Fig. 15. Despite the shorter stationary part of the trajectory, the shaking feature appears, again and again, almost periodically. The orbit does not repeat itself at each of the shaking parts of the evolution, thus confirming the fact that many frequencies are involved in this dynamics. In fact, with

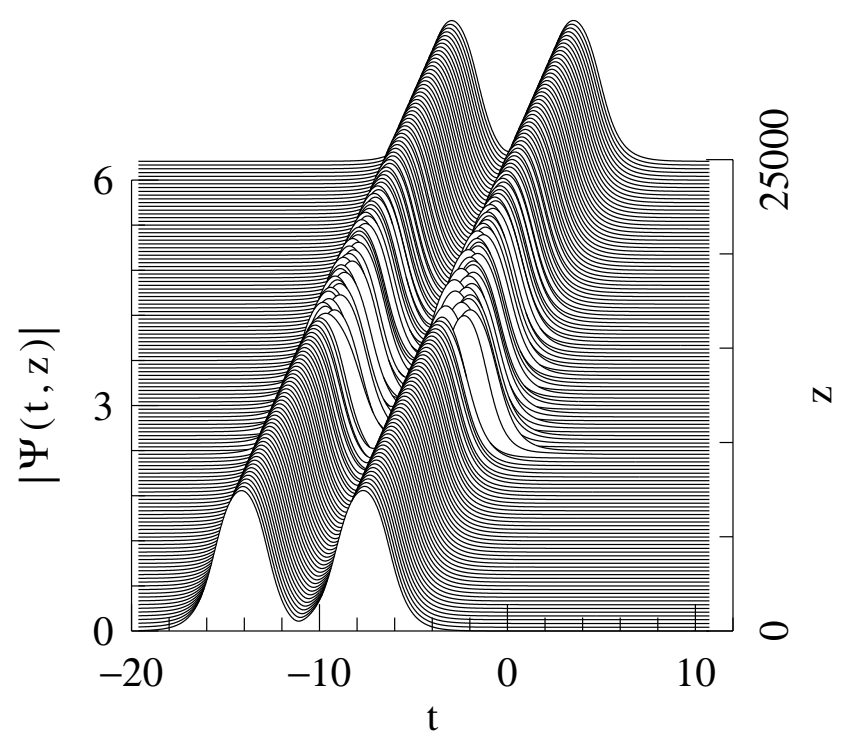

Fig. 14. Evolution of the pulse profile of the shaking soliton pair shown in Fig. 13.

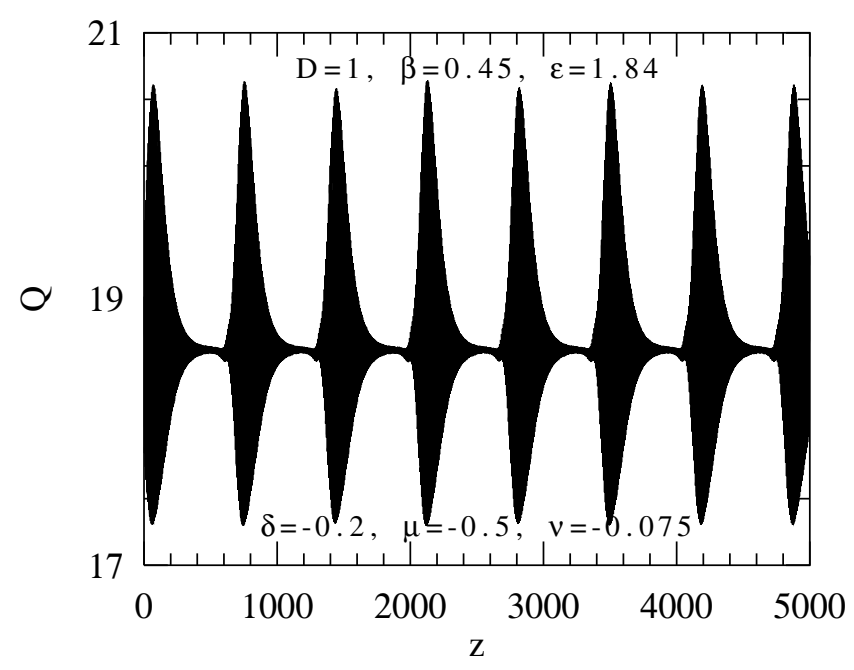

Fig. 15. Another example of a spontaneously shaken soliton pair. The parameters of the simulation are written inside the figure. This example shows that the shaking feature can occur for a relatively wide range of the system parameters.

a slight change in the values of the equation parameters, the differences between the cycles can be made considerably larger, showing that the chaotic nature of the motion becomes more pronounced.

The peak amplitudes, $A_{1}$ and $A_{2}$, of the two solitons in the pair for one cycle of evolution are shown in Fig. 16. The two amplitudes have almost the same value during the near-stationary part of the evolution. They start to oscillate due to the instability, but the amplitudes of oscillation are clearly different. When the instability is over, the

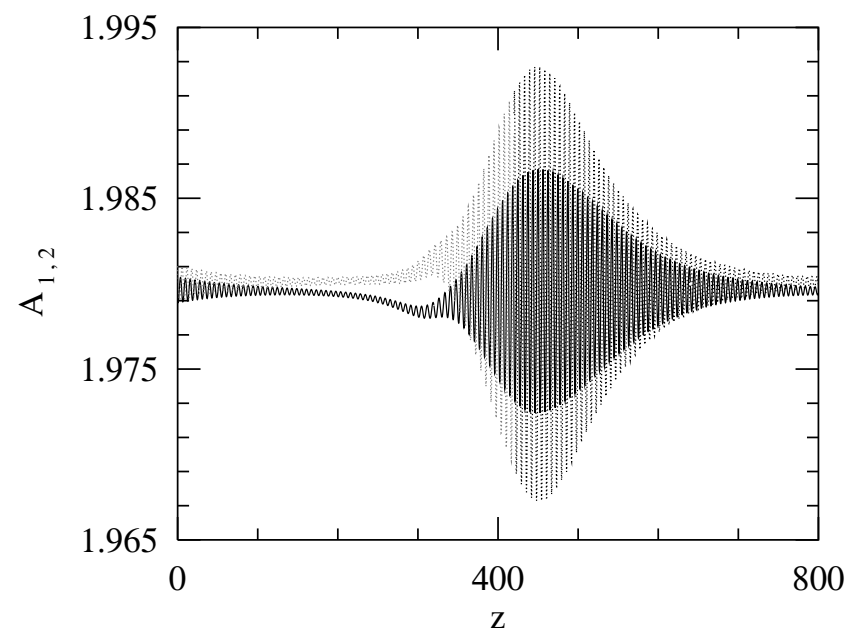

Fig. 16. Peak amplitudes of the two solitons forming a shaking pair. Gray-dotted line is for $A_{1}$, and black-solid line is for $A_{2}$. Only about one cycle of the spontaneous shaking represented in Fig. 15 is shown here, with a higher resolution along the $z$ direction. 
oscillations decay and the soliton pair becomes nearly stationary again. The soliton pair is slightly asymmetric in that the right-hand side (r.h.s.) pulse (gray dotted line) has an average amplitude which is larger than that of the pulse on the left (solid line). This asymmetry also comes from the phase asymmetry, as previously discussed.

\section{Dissipative Solitons in Higher Dimensions}

\section{1. (2+1)-dimensional dissipative solitons}

For two transverse dimensions, it was also found that there are at least two sets of stable stationary localized solutions with a major qualitative difference: one set consists of "self-focusing" beams (solitons) and the other set of "self-defocusing" beams (anti-solitons [Ankiewicz et al., 2008]). When we smoothly change the parameters of the medium, viz. the external pump, nonlinearity, spectral filtering, etc., the solutions also change smoothly in a given range, but abrupt changes occur at the bifurcation points. One type of soliton cannot be transformed into the other - either smoothly or through a bifurcation. In fact, they are separated in parameter space by a region where no solitons exist. There is no way of continuously transforming a soliton from one region into a soliton of the other region.

In the (2+1)-dimensional case, the complex Ginzburg-Landau equation describing beam propagation reads [Ankiewicz et al., 2008]:

$$
\begin{aligned}
i \psi_{z} & +\frac{1}{2} \nabla_{\perp}^{2} \psi+|\psi|^{2} \psi+\nu|\psi|^{4} \psi \\
& =i \delta \psi+i \epsilon|\psi|^{2} \psi+i \beta \nabla_{\perp}^{2} \psi+i \mu|\psi|^{4} \psi,
\end{aligned}
$$

where $\psi(z, r)$ is the normalized envelope of the field, $\nabla_{\perp}^{2}$ is the transverse Laplacian, $z$ is the propagation distance, $\nu$ is the coefficient of the quintic nonlinearity, $\delta$ represents linear losses, $\epsilon$ is the nonlinear gain coefficient, $\beta$ stands for spectral angular filtering of the cavity, and $\mu$ characterizes the saturation of the nonlinear gain. This equation is applicable to wide aperture lasers [Meucci et al., 1998; Fedorov et al., 2003] and vertical external cavity semiconductor devices [Mercier \& Moloney, 2002]. It can also be applied to multimode optical fibers made of erbium doped glass, when the number of modes is very large.

There are no known analytic solutions of Eq. (2). The only way to obtain solutions is through numerical simulations. The latter requires a large amount of computer time. To have an idea of how the solution may depend on the parameters of the equation requires a vast amount of simulations. We are interested in finding the regions in the space of parameters of Eq. (2) which admit stable soliton solutions. Before starting simulations, it would be nice to have some estimates of where, in the parameter space, these solutions could be found. For that purpose, approximate techniques can be very helpful, since they serve as a guide for our numerical simulations. Thus, some simplification is a necessity.

In order to approximate the beam shape, a higher-order radially-symmetric Gaussian-type function can be used as a trial function, viz.

$$
\begin{aligned}
\psi(r, z)= & \frac{\sqrt{2}}{\pi^{3 / 4} f(z)} \exp \left[-\frac{r^{4}}{2 m^{2} f^{4}(z)}-\frac{r^{2}}{f^{2}(z)}\right] \\
& \times \sqrt{\frac{e^{-m^{2}} Q(z)}{m \operatorname{erfc}(m)}} \exp \left[i\left(r^{2} c(z)-\theta(z)\right)\right],
\end{aligned}
$$

where $r$ is the radial co-ordinate, $m$ is a parameter that controls the relative contribution of each term $\left(r^{2}\right.$ and $\left.r^{4}\right)$ in the trial function. The complementary error function, $\operatorname{erfc}(m)$, appears in $(3)$ to give the correct normalization. Henceforth, we consider $m$ to be a constant. The function $\theta(z)$ in (3) represents the axial phase evolution. $f(z)$ and $c(z)$ are two unknown functions to fit Eq. (3) to the actual solution of the CGLE using approximate methods. For a stationary solution, the values $f, c$ and $Q$ are constants and $\theta^{\prime}(z)=\omega=$ const., so that the phase evolution is linear, $\theta(z)=\omega z$. For pulsating solutions with radial symmetry, $\omega=\omega(z)$ oscillates rather than being constant. Our studies have shown that the function (3) gives a good fit for the beam shape as well as for the regions of existence of solitons in the space of the equation parameters.

Usually, the solutions are radially symmetric. Two examples illustrating the two types of solitons numerically found are shown in Figs. 17 and 18. The amplitude profiles are qualitatively similar. However, the phase profiles clearly show that the chirps are of opposite sign. Thus, these two examples belong to different classes of solitons. We also notice the different phase scales in these two figures - the positive chirp is much stronger than the negative one. The amplitude scales show that they may carry more power. These qualitative observations are in full agreement with the predictions of the low-dimensional approximation. 


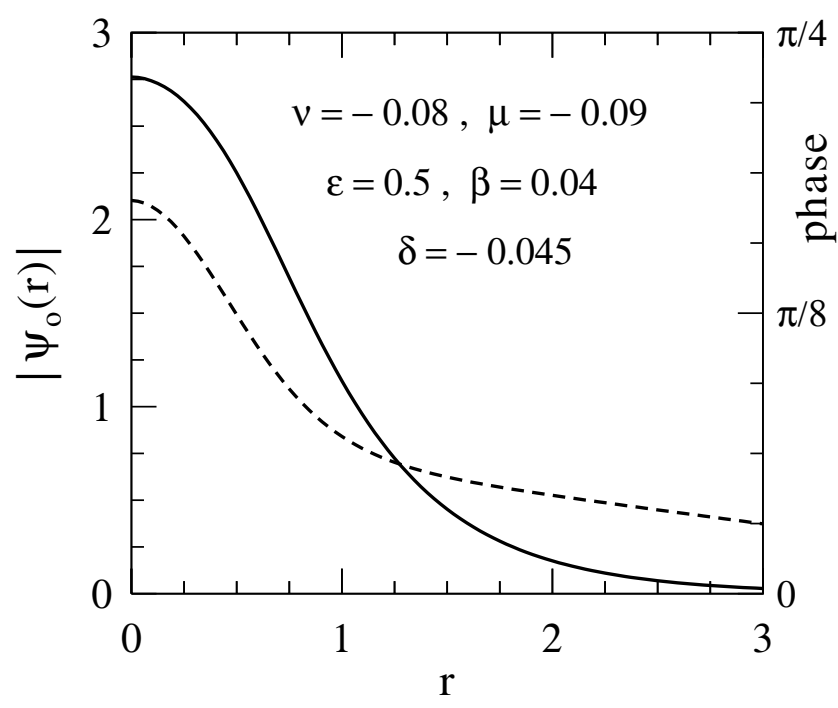

Fig. 17. Amplitude (solid line) and phase (dashed line) profiles of the soliton solution obtained for the following values of the parameters: $\beta=0.04, \nu=-0.08, \delta=-0.045, \epsilon=0.5$ and $\mu=-0.09$.

By solving either of the dynamical systems for various values of the equation parameters of the CGLE, we can construct regions of stable fixed points. As before, and in order to keep the presentation of the results reasonably simple, we keep three equation parameters fixed and change the other two, looking at these two-dimensional slices of the five-dimensional space of the equation parameters $(\beta, \epsilon, \delta, \mu$ and $\nu)$ to find where stationary solutions exist and are stable. Proceeding in this way, we have observed the existence of two separate regions of stable fixed points. As in the $(1+1)-\mathrm{D}$ case, a point from one region cannot be transformed into a point from the other region with a continuous change of parameters. Thus, it appears that these two regions correspond to two different types of solitons of the CGLE. One of the branches has positive chirp, $c$, and high power, $Q$, while the other one has negative chirp and low power. Within the lowdimensional approximation, the fixed points (FPs) in both regions are stable.

Figures 19 and 20 respectively show two 2-D regions where these two types of different solutions exist. In particular, Fig. 19 shows the region of existence of solitons with negative chirp. The negative chirp means that the beam is continuously self-focusing. Figure 20 shows the region of existence of solitons with positive chirp. Such a beam is continuously self-defocusing. The gray area in each case represents the results obtained from the lowdimensional approach, while the hatched area is

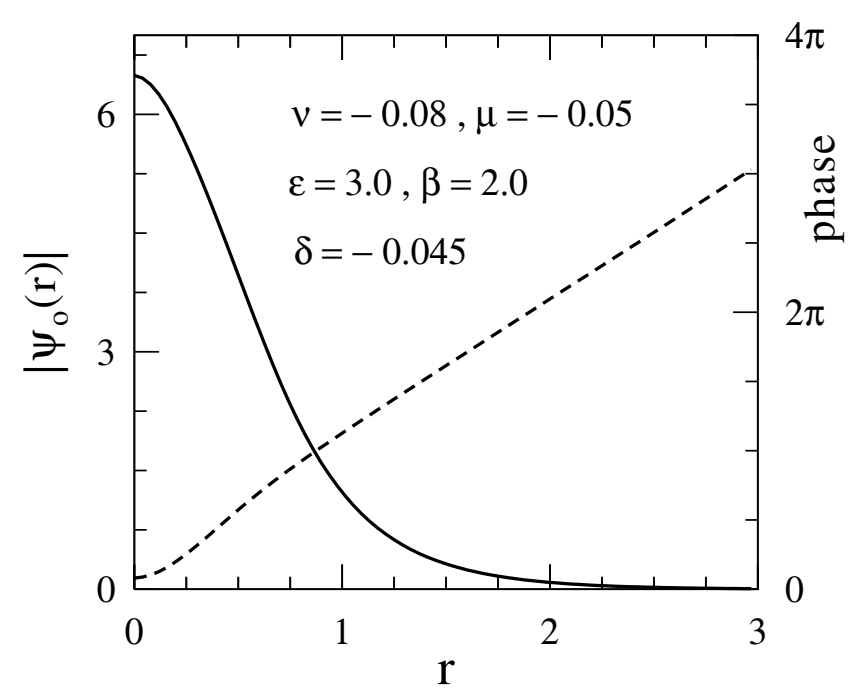

Fig. 18. Amplitude (solid line) and phase (dashed line) profiles of the anti-soliton with $\epsilon=3, \beta=2$.

obtained from numerical simulations of the whole CGLE, as described above. The region obtained with the reduced model approximates the exact results quite well in the case of self-focusing solitons. The agreement is qualitatively reasonable for the region of self-defocusing beams (anti-solitons). A similar situation has been observed in the 1-D case [Ankiewicz et al., 2007].

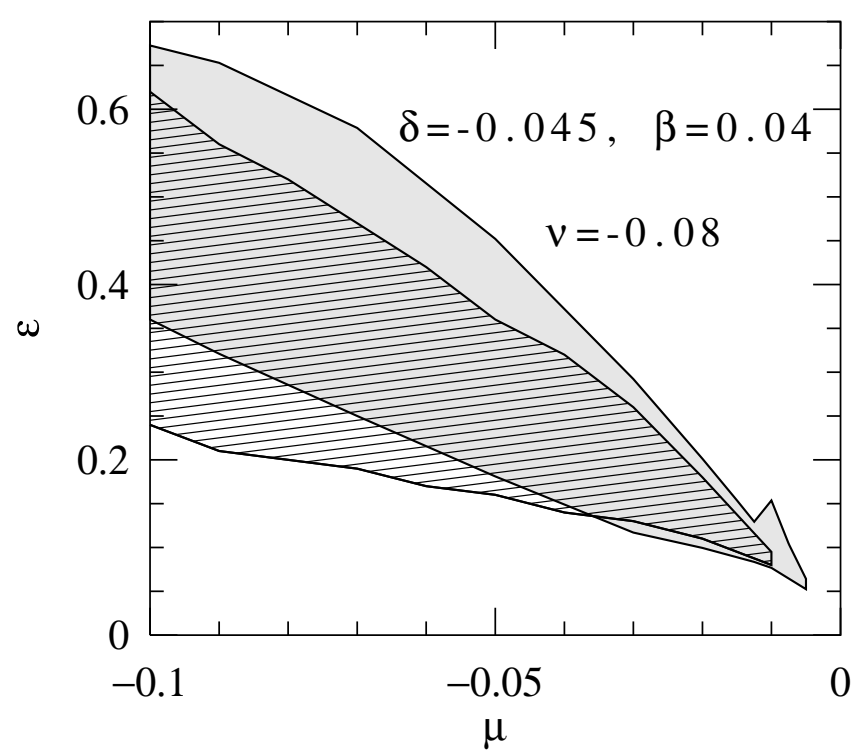

Fig. 19. Region of existence of solitons with negative chirp $\left(c_{0}<0\right)$ in the $(\mu, \epsilon)$ plane found using the trial function (3). Stable FPs exist in the gray region. The hatched area shows the region where stable stationary solutions are obtained numerically using the full Ginzburg-Landau equation. The two regions match reasonably well. 


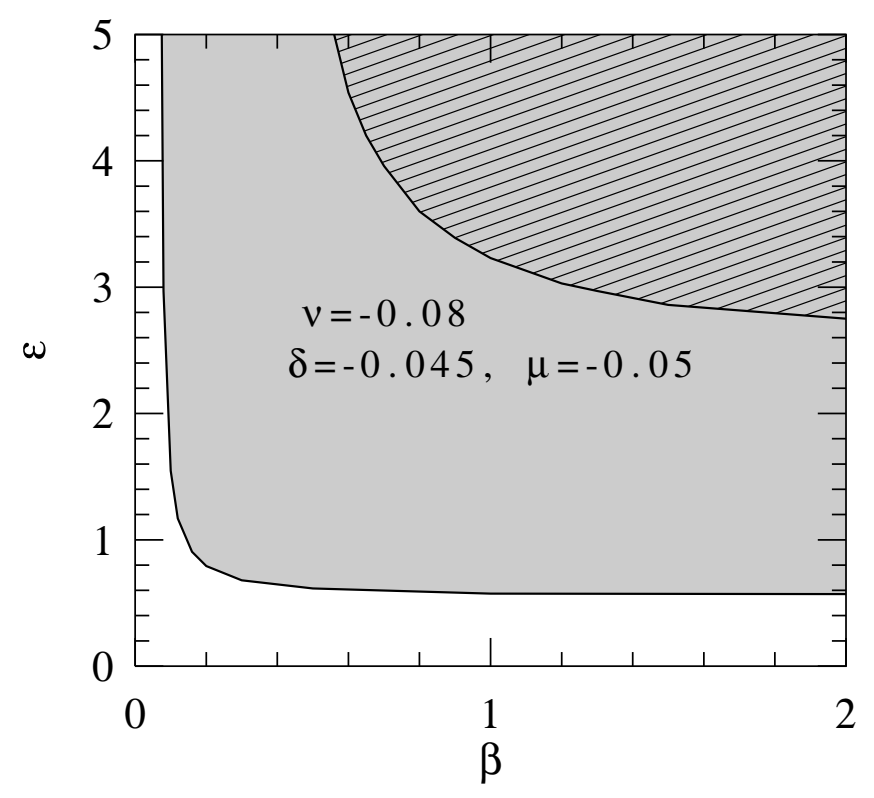

Fig. 20. Region of existence of anti-solitons with positive chirp $\left(c_{0}>0\right)$ (or self-defocusing type of solitons) in the $(\beta, \epsilon)$ plane. The gray region is obtained with the low-dimensional approximation while the hatched region is obtained with direct numerical simulations. The parameters are: $\nu=-0.08, \mu=-0.05, \delta=-0.045$.

Clearly, in the case of a conservative medium, the beam could not be stationary when either selffocusing or self-defocusing occurs. In dissipative media, this effect, involving an internal radial flux of energy in the beam, produces the dynamical equilibrium within the stationary solution. Thus, the beam can be either continuously self-focusing or continuously self-defocusing.

When the radially-symmetric solution loses stability, it may be transformed into a more complicated beam. Direct simulations reveal that, in addition to stationary beams, there are localized structures that cannot easily be obtained using simple approximations. The regions of existence of regular solitons or antisolitons are surrounded by regions of nonstationary beams. A rich variety of such solutions exists, with unique dynamics for each type. In particular, 2D exploding solitons are also observed close to the boundaries of the region of existence of antisolitons, as happened in the 1D case, but with its own specificities.

\section{2. (3+1)-dimensional dissipative solitons}

In the $(3+1) D$ spatiotemporal case, the CGLE master equation can include chromatic dispersion and spectral filtering from Eq. (1), as well as spatial diffraction from Eq. (2), resulting in:

$$
\begin{aligned}
i \psi_{z} & +\frac{D}{2} \psi_{t t}+\frac{1}{2} \psi_{x x}+\frac{1}{2} \psi_{y y}+|\psi|^{2} \psi+\nu|\psi|^{4} \psi \\
& =i \delta \psi+i \epsilon|\psi|^{2} \psi+i \beta \psi_{t t}+i \mu|\psi|^{4} \psi
\end{aligned}
$$

Localized stable optical soliton solutions of Eq. (3) can be called dissipative light bullets. They can also be considered as extensions of "cavity solitons". Again, stable formations can be found provided the system parameters are chosen in specific regions. Recently, the existence of stable light bullets in both regimes of chromatic dispersion was proven numerically, as well as more complicated pulsating dynamics. It seems, in general, that dissipative soliton solutions tolerate an increase of the spatiotemporal dimensionality of the problem quite well. This means that similar dynamical behavior, such as stationary light bullets and pulsating LB solutions can be found widely. A review of recent results presenting a variety of dissipative light bullets solutions, i.e. stationary, pulsating solutions and complexes, can be found in [Akhmediev et al., 2007]. Although the tools of analysis used in 1D can be extrapolated to higher dimensions, the task of computing exact solutions becomes difficult, so that the help of approximate semi-analytical methods becomes useful in mapping domains of existence for each type of solution [Kamagate et al., 2008]. Naturally the variety of solutions should be much larger in $(3+1) \mathrm{D}$, especially for pulsations and complexes [Veretenov et al., 2008] which is an open field of research.

\section{Conclusions}

Dissipative solitons form a new paradigm for the investigation of phenomena involving stable structures in nonlinear systems far from equilibrium. We have reviewed recent results in the area of dissipative solitons of dynamical systems governed by the CGLE.

In particular, we reported on the existence of two kinds of stationary stable single solitons with very distinct characteristics — we call them "solitons" and "antisolitons". They have opposite chirp signs and hence opposite directions of internal flux of energy; their energy values also differ considerably from each other. Generally pulsations appear at the boundaries of the region of existence of solitons, whereas exploding solitons can be observed at the antisoliton boundaries. 
Another interesting phenomenon that we found is "dissipative soliton resonance" when the energy of the soliton can increase indefinitely at certain values of the system parameters. This effect can find applications in the design of passively modelocked lasers generating high-energy ultra-short pulses.

The interaction of dissipative solitons is an extensive field of research in itself. It can be quite different from the interaction of conservative solitons. In particular, the interaction of two single stationary 1-D stable solutions can generate new solutions with characteristics which are very different from those of their two single components. These solutions can be stable, vibrating or even chaotic.

Dynamical systems with higher dimensionality create a larger variety of dissipative solitons. Studies in this area are really at the initial stage of research.

\section{Acknowledgments}

N. Akhmediev and A. Ankiewicz acknowledge support from the Australian Research Council Discovery Projects funding scheme (project number DP0663216). The work of J. M. Soto-Crespo was supported by the M. E. C. under contract FIS2006-03376. Ph. Grelu was supported by Agence Nationale de la Recherche (project ANR05-BLAN0152-01).

\section{References}

Akhmediev, N. \& Ankiewicz, A. [1997] Solitons, Nonlinear Pulses and Beams (Chapman \& Hall, London).

Akhmediev, N., Ankiewicz, A. \& Soto-Crespo, J. M. [1997] "Multisoliton solutions of the complex Ginzburg-Landau equation," Phys. Rev. Lett. 79, 4047-4050.

Akhmediev, N., Soto-Crespo, J. M. \& Town, G. [2001] "Pulsating solitons, chaotic solitons, period doubling, and pulse coexistence in mode-locked lasers: Complex Ginzburg-Landau equation," Phys. Rev. E 63, 056602 .

Akhmediev, N. \& Soto-Crespo, J. M. [2003] "Exploding solitons and Shil'nikov's Theorem," Phys. Lett. A 317, 287-292.

Akhmediev, N. \& Ankiewicz, A. (eds.) [2005] Dissipative Solitons, Lecture Notes in Physics, Vol. 661 (Springer, Berlin, Heidelberg).

Akhmediev, N., Soto-Crespo, J. M., Grapinet, M. \& Grelu, Ph. [2005] "Dissipative soliton interactions inside a fiber laser cavity," Opt. Fib. Technol. 11, 209228.
Akhmediev, N., Soto-Crespo, J. M. \& Grelu, Ph. [2007] "Spatio-temporal optical solitons in nonlinear dissipative media: From stationary light bullets to pulsating complexes," Chaos 17, 037112.

Akhmediev, N. \& Ankiewicz, A. (eds.) [2008] Dissipative Solitons: From Optics to Biology and Medicine, Lecture Notes in Physics, Vol. 751 (Springer, Berlin, Heidelberg).

Akhmediev, N., Soto-Crespo, J. M. \& Grelu, Ph. [2008] "Roadmap to ultra-short record high-energy pulses out of laser systems," Phys. Lett. A 372, 31243128.

Ankiewicz, A., Devine, N., Akhmediev, N. \& SotoCrespo, J. M. [2007] "Dissipative solitons and antisolitons," Phys. Lett. A 370, 454-458.

Ankiewicz, A., Devine, N., Akhmediev, N. \& SotoCrespo, J. M. [2008] "Continuously self-focusing and continuously self-defocusing two-dimensional beams in dissipative media," Phys. Rev. A 77, 033840.

Aranson, I. \& Kramer, L. [2002] "The world of the complex Ginzburg-Landau equation," Rev. Mod. Phys. 74, 99-143.

Chong, A., Renninger, W. H. \& Wise, F. W. [2008] "Properties of normal-dispersion femtosecond fiber lasers," J. Opt. Soc. Am. B 25, 140-148.

Cundiff, S. T., Soto-Crespo, J. M. \& Akhmediev, N. [2002] "Experimental evidence for soliton explosions," Phys. Rev. Lett. 88, 073903.

Deissler, R. J. \& Brand, H. [1994] "Periodic, quasiperiodic and chaotic localized solutions of the quintic complex Ginzburg-Landau equation," Phys. Rev. Lett. 72, 478-481.

Descalzi, O. [2003] "On the stability of localized structures in the complex Ginzburg-Landau equation," Physica A 327, 23-28.

Descalzi, O. \& Brand, H. R. [2005] "Stable stationary and breathing holes at the onset of a weakly inverted instability," Phys. Rev. E 72, 055202.

Descalzi, O., Düring, G. \& Tirapegui, E. [2005] "On the stable hole solutions in the complex GinzburgLanadau equation," Physica A 356, 66-71.

Fedorov, S. V., Rosanov, N. N., Shatsev, A. N., Veretenov, N. A. \& Vladimirov, A. G. [2003] "Topologically multicharged and multihumped rotating solitons in wide-aperture lasers with a saturable absorber," IEEE J. Quant. Electron. 39, 197-205.

Fernandez, A., Fuji, T., Poppe, A., Fürbach, A., Krausz, F. \& Apolonski, A. [2004] "Chirped-pulse oscillators: A route to high-power femtosecond pulses without external amplification," Opt. Lett. 29, 1366-1368.

Grapinet, M. \& Grelu, Ph. [2006] "Vibrating soliton pairs in a mode-locked laser cavity," Opt. Lett. 31, 2115-2117.

Grelu, Ph., Belhache, F., Gutty, F. \& Soto-Crespo, J. M. [2002] "Phase-locked soliton pairs in a stretched-pulse fiber laser," Opt. Lett. 27, 966-968. 
Grelu, Ph., Belhache, F., Gutty, F. \& Soto-Crespo, J. M. [2003] "Relative phase locking of pulses in a passively mode-locked fiber laser," J. Opt. Soc. Am. B 20, 863-870.

Grelu, Ph. \& Akhmediev, N. [2004] "Group interactions of dissipative solitons in a laser cavity: The case of 2+1," Opt. Express 12, 3184-3189.

Grelu, Ph. \& Soto-Crespo, J. M. [2004] "Multisoliton states and pulse fragmentation in a passively modelocked fibre laser," J. Opt. B: Quant. Semiclass. Opt. 6, S271-S278.

Haus, H. A. [2000] "Mode-locking of lasers," IEEE J. Sel. Top. Quant. Electron. 6, 1173-1185.

Leblond, H., Komarov, A., Salhi, M., Haboucha, A. \& Sanchez, F. [2006] "Cis bound states of three localized pulses of the cubic-quintic CGL equation," J. Opt. A 8, 319-326.

Mercier, J.-F. \& Moloney, J. V. [2002] "Derivation of semiconductor laser mean-field and Swift-Hohenberg equations," Phys. Rev. E 66, 036221.

Meucci, R., Labate, A., Ciofini, M. \& Wang, P.-Y. [1998] "Bifurcations of hexagonal patterns stabilized and selected with spatial perturbations in a wide-aperture laser," Quant. Semiclass. Opt. 10, 803-808.

Nicolis, G. \& Prigogine, I. [1977] Self-Organization in Nonequilibrium Systems - From Dissipative Structures to Order Through Fluctuations (Wiley, NY).

Olivier, M., Roy, V., Piché, M. \& Babin, F. [2004] "Pulse collisions in the stretched-pulse fiber laser," Opt. Lett. 29, 1461-1463.
Renninger, W. H., Chong, A. \& Wise, F. W. [2008] "Dissipative solitons in normal-dispersion fiber lasers," Phys. Rev. A 77, 023814 (1-4).

Salhi, M., Haboucha, A., Leblond, H. \& Sanchez, F. [2008] "Theoretical study of figure-eight all-fiber laser," Phys. Rev. A 77, 033828 (1-9).

Soto-Crespo, J. M., Akhmediev, N., Afanasjev, V. V. \& Wabnitz, S. [1997] "Pulse solutions of the cubicquintic complex Ginzburg-Landau equation in the case of normal dispersion," Phys. Rev. E 55, 47834796.

Soto-Crespo, J. M., Akhmediev, N. \& Ankiewicz, A. [2000] "Pulsating, creeping, and erupting solitons in dissipative systems," Phys. Rev. Lett. 85, 2937-2940.

Soto-Crespo, J. M., Akhmediev, N. \& Chiang, K. [2001] "Simultaneous existence of a multiplicity of stable and unstable solitons in dissipative systems," Phys. Lett. A 291, 115-123.

Soto-Crespo, J. M., Grelu, Ph., Akhmediev, N. \& Devine, N. [2007] "Soliton complexes in dissipative systems: Vibrating, shaking and mixed soliton pairs," Phys. Rev. E 75, 016613.

van Saarlos, W. \& Hohenberg, P. C. [1990] "Pulses and fronts in the complex Ginzburg-Landau equation near a subcritical bifurcation," Phys. Rev. Lett. 64, 749.

Wise, F. W., Chong, A. \& Renninger, W. H. [2008] "High-energy femtosecond fiber lasers based on pulse propagation at normal dispersion," Laser Photon. Rev. 2, 58-73. 